$\xi=$ 臬

\title{
Non-collinear libration points in CR3BP when less massive primary is an heterogeneous oblate body with $\mathbf{N}$-layers
}

\author{
M. Javed Idrisi ${ }^{1 *}$, Kumari Shalini ${ }^{2}$ \\ ${ }^{1}$ Department of Mathematics, Al-Falah University, Faridabad (Haryana) -121004, India \\ ${ }^{2}$ Department of Mathematics, Deshbandhu College, University of Delhi, Kalkaji, New Delhi -110017, India \\ *Corresponding authorE-mail:mjavedidrisi@gmail.com
}

\begin{abstract}
In the present paper, the existence of non-collinear libration points has been shown in circular restricted three-body problem when less massive primary is a heterogeneous oblate body with N-layers. Further, the stability of non-collinear libration points is investigated in linear sense and found that the non-collinear libration points are stable for the critical value of mass parameter $\mu \leq \mu_{\text {crit }}=\mu_{\mathrm{o}}-3.32792 k_{1}$ $-1.16808 k_{2}$.
\end{abstract}

Keywords: Celestial Mechanics; Restricted Three-Body Problem; Libration Points; Stability; Heterogeneous Oblate Spheroid.

\section{Introduction}

The restricted problem of three-body describes the motion of infinitesimal mass moving in the gravitational field of two massive primaries in the same plane or out of plane called two dimensional or three dimensional problem accordingly. The primaries are revolving around their center of mass either in circular or elliptical orbits under the influence of their mutual gravitational attraction. If the orbit of the primaries around their center of mass is elliptic, problem is said to be elliptic restricted three-body problem (ER3BP or ERTBP) and if the orbit of the primaries around their center of mass is circular, problem is said to be circular restricted three-body problem or restricted three-body problem, denoted by CR3BP or CRTBP or RTBP or R3BP.

The problem possesses five equilibrium points out of which three are collinear and two non-collinear. The collinear libration points are unstable while non-collinear are stable for the mass ratio $\mu \leq$ 0.038520896505 [2].Some studies related to the equilibrium points in R3BP or ER3BP, taken into account the oblateness and triaxiality of the primaries, Coriolis and Centrifugal forces, variation of the masses of the primaries and the infinitesimal mass etc. are discussed by Danby [1]; Szebehely [2]; Vidyakin [3]; Sharma [4]; Subbarao and Sharma [5]; Sharma et.al. [6]; Choudhary R. K. [7]; Bhatnagar and Hallan [8]; Cid R. et. al. [9]; El-Shaboury [10]; Bhatnagar et al. [11]; Selaru D. et.al. [12]; Markellos et al. [13]; Subbarao and Sharma [14]; Khanna and Bhatnagar [15], [16]; Roberts G.E. [18]; Oberti and Vienne [19]; Sosnytskyi [20]; Perdiouet. al. [21]; Arredondo et.al. [22]; Idrisi and Taqvi [23]; Idrisi [24]; Idrisi and Amjad [25], Idrisi [26].

We got the idea of our problem from the paper 'Rotating Stratified Heterogeneous Oblate Spheriodin Newtonian Physics' by Esteban and Vazquez. [17]. in their paper they have taken three layers in a stratified non-conformal heterogeneous oblate spheroidal system. We wish to extend this study to the restricted three body problem.

\section{Equations of motion}

Let $m_{1}, m_{2}$ and $m_{3}$ be the masses of more massive primary, less massive primary and infinitesimal mass respectively. We consider the less massive primary $m_{2}$ as an heterogeneous oblate body with N-layers having different densities $\rho_{i}$ and axes $a_{i}, b_{i}$ and $c_{i}$ such that $\rho_{i+1}>\rho_{i}, a_{i+1}>a_{i}, b_{i+1}>b_{i}, c_{i+1}=c_{i}, a_{i}=b_{i}>c_{i}, i=1,2, \ldots, \mathrm{N}$. The primaries are revolving with angular velocity $n$ in circular orbits about their common centre of mass $O$ and $m_{3}$ is moving under the gravitational field of $m_{1}$ and $m_{2}$ in the same plane. The line joining $m_{1}$ and $m_{2}$ is taken as $\mathrm{X}$ - axis and ' $O$ ' their center of mass as origin and the line passing through $O$ and perpendicular to $O X$ and lying in the plane of motion of $m_{1}$ and $m_{2}$ is the $Y$-axis. We consider a synodic system of coordinates $O(x y z)$; initially coincident with the inertial system $O(X Y Z)$, rotating with the angular velocity $n$ about $Z$-axis (the $z$-axis is coincident with $Z$-axis). The distances of $m_{3}$ from $m_{j}$ and $\mathrm{O}$ are $\boldsymbol{r}_{j}$ and $\boldsymbol{r}$ respectively, $j=1$, 2. Our aim is to find the equations of motion of $m_{3}$ using the terminology of Szebehely (1967) in the synodic co-ordinate system and dimensionless variables i.e. the distance between the primaries $m_{1}$ and $m_{2}$ is unity, the unit of time $t$ is such that the gravitational constant $G=1$ and the sum of the masses of the primaries is unity i.e. $m_{1}+m_{2}=1$.

The equations of motion of the infinitesimal mass $m_{3}$ in the synodic coordinate system and dimensionless variables are given by

$$
\begin{aligned}
& \ddot{x}-2 n \dot{y}=\Omega_{x}, \\
& \ddot{y}+2 n \dot{x}=\Omega_{y},
\end{aligned}
$$

where

$$
\begin{aligned}
\Omega= & \frac{n^{2}}{2}\left[(1-\mu) r_{1}^{2}+\mu r_{2}^{2}\right]+\frac{1-\mu}{r_{1}}+ \\
& \frac{\mu}{r_{2}}+\frac{k_{1}}{2 r_{2}^{3}},
\end{aligned}
$$




$$
\begin{aligned}
& \Omega_{x}=n^{2} x-(1-\mu) \frac{(x-\mu)}{r_{1}^{3}}-\mu \frac{(x+1-\mu)}{r_{2}^{3}}- \\
& \frac{3 k_{1}(x+1-\mu)}{2 r_{2}^{5}}, \\
& \Omega_{y}=y\left[n^{2}-\frac{(1-\mu)}{r_{1}^{3}}-\frac{\mu}{r_{2}^{3}}-\frac{3 k_{1}}{2 r_{2}^{5}}\right] \text {, } \\
& n^{2}=1+\frac{3}{2} k_{2} \text {, Is the mean motion of the primaries, } \\
& k_{1}=\frac{4 \pi}{3} \sum_{i=1}^{N} a_{i}^{2} c_{i}\left(\rho_{i}-\rho_{i+1}\right) \sigma_{i}, \\
& k_{2}=\sum_{i=1}^{n} \sigma_{i}, \\
& \sigma_{i}=\frac{a_{i}^{2}-c_{i}^{2}}{5}, \\
& r_{1}^{2}=(x-\mu)^{2}+y^{2}, \\
& r_{2}^{2}=(x+1-\mu)^{2}+y^{2}, \\
& \mu=\frac{m_{2}}{m_{1}+m_{2}}<\frac{1}{2} \\
& \Rightarrow m_{1}=1-\mu \text {; } \\
& m_{2}=\mu=\frac{4 \pi}{3} \sum_{i=1}^{N} a_{i}^{2} c_{i}\left(\rho_{i}-\rho_{i+1}\right) .
\end{aligned}
$$

\section{Non-collinear libration points}

At the libration points all the derivatives of $n^{\text {th }}$ order are zero, therefore the equations of motion (1) becomes

$$
\begin{aligned}
& n^{2} x-(1-\mu) \frac{(x-\mu)}{r_{1}^{3}}-\mu \frac{(x+1-\mu)}{r_{2}^{3}}- \\
& \frac{3 k_{1}(x+1-\mu)}{2 r_{2}^{5}}=0
\end{aligned}
$$

and

$$
y\left[n^{2}-\frac{(1-\mu)}{r_{1}^{3}}-\frac{\mu}{r_{2}^{3}}-\frac{3 k_{1}}{2 r_{2}^{5}}\right]=0 .
$$

The collinear libration points are the solution of the Equations (5) and (6) for $\mathrm{y} \neq 0$ i.e.

$$
\begin{aligned}
& n^{2} x-(1-\mu) \frac{(x-\mu)}{r_{1}^{3}}-\mu \frac{(x+1-\mu)}{r_{2}^{3}}- \\
& \frac{3 k_{1}(x+1-\mu)}{2 r_{2}^{5}}=0
\end{aligned}
$$

and

$$
n^{2}-\frac{(1-\mu)}{r_{1}^{3}}-\frac{\mu}{r_{2}^{3}}-\frac{3 k_{1}}{2 r_{2}^{5}}=0 .
$$

On substituting $k_{1}=0$ and $k_{2}=0$, the solution of Eqns. (7) and (8) is $r_{1}=1, r_{2}=1$ and from Eqn. (2), $n=1$.

Now we assume that the solution of Eqns. (7) and (8) for $k_{1} \neq 0, k_{2}$ $\neq 0$ as

$r_{1}=1+\xi_{1}, r_{2}=1+\xi_{2}, \xi_{1}, \xi_{2}<<1$.

Substituting these values of $r_{1}$ and $r_{2}$ in the Eqns. (3) and (4), we get

(2) $x=\mu-\frac{1}{2}+\xi_{2}-\xi_{1}$

$y= \pm \frac{\sqrt{3}}{2}\left[1+\frac{2}{3}\left(\xi_{2}+\xi_{1}\right)\right]$

Now, substituting the values of $x, y$ from Eqns. (9) and $r_{1}=1+\xi_{1}$, $r_{2}=1+\xi_{2}$ in the Eqns. (7) and (8) and neglecting higher order terms, we obtain

$$
\begin{aligned}
& \xi_{1}=\left[\frac{1}{2}-\frac{\mu}{(1-\mu)}\right]\left(k_{1}+k_{2}\right), \\
& \xi_{2}=\left[1+\frac{\mu}{(1-2 \mu)}\right] k_{1}-\frac{\mu}{(1-2 \mu)} k_{2} .
\end{aligned}
$$

Thus the coordinates of the non-collinear libration points $L_{4,5}$ are

$$
\begin{gathered}
x=\mu-\frac{1}{2}+\left[\frac{\mu}{1-\mu}+\frac{\mu}{1-2 \mu}\right] k_{1}- \\
{\left[1-\frac{\mu}{1-\mu}+\frac{\mu}{1-2 \mu}\right] k_{2},} \\
y= \pm \frac{\sqrt{3}}{2}\left[1+\frac{2}{3}\left\{\begin{array}{l}
\left(2-\frac{\mu}{1-\mu}+\frac{\mu}{1-2 \mu}\right) k_{1}+ \\
\left(1-\frac{\mu}{1-\mu}-\frac{\mu}{1-2 \mu}\right) k_{2}
\end{array}\right\}\right]
\end{gathered}
$$

\section{Stability of non-collinear libration points}

The equations of the motion of the infinitesimal mass are

$$
\begin{aligned}
& \ddot{x}-2 n \dot{y}=\Omega_{x}, \\
& \ddot{y}+2 n \dot{x}=\Omega_{y} .
\end{aligned}
$$

To study the possible motion of the infinitesimal mass around the libration points let the coordinates of these points are $\left(x_{0}, y_{0}\right)$. If we give small displacement $(\zeta, \eta)$ to $\left(x_{0}, y_{0}\right)$ and considering only linear terms in $\zeta$ and $\eta$, the variation $\zeta$ and $\eta$ can be written as: $\zeta=$ $x-x_{0}$ and $\eta=y-y_{0}$ and the equations of the motion become

$$
\begin{gathered}
\ddot{\varsigma}-2 n \dot{\eta}=\Omega_{x}\left(x_{0}+\varsigma, y_{0}+\eta\right)=\varsigma \Omega_{x x}^{o}+\eta \Omega_{x y}^{o}, \\
\ddot{\eta}+2 n \dot{\varsigma}=\Omega_{y}\left(x_{0}+\varsigma, y_{0}+\eta\right)=\varsigma \Omega_{y x}^{o}+\eta \Omega_{y y}^{o} .
\end{gathered}
$$

where ' $\mathrm{o}$ ' indicates that the partial derivatives are to be calculated at the libration points under consideration.

The characteristic equation of the Equations (12) is given by

$$
\left.\lambda^{4}+\left(4 n^{2} \stackrel{\stackrel{o}{\Omega}}{\Omega_{x x}} \stackrel{\stackrel{o}{\Omega}}{\Omega_{y y}}\right) \lambda^{2}+\stackrel{o}{\Omega_{x x}} \stackrel{o}{\Omega} y y-\stackrel{o}{\Omega} x y\right)^{2}=0
$$

where 


$$
\begin{aligned}
& \Omega_{x x}=\frac{3}{4}+\left(-\frac{3 \mu}{8}-\frac{3 \mu}{1-2 \mu}-\frac{9 \mu}{4(1-\mu)}\right) k_{1}+ \\
& \left(\frac{33}{8}-\frac{45}{8} \mu+\frac{3 \mu}{1-2 \mu}-\frac{9 \mu}{4(1-\mu)}\right) k_{2} \text {, } \\
& \stackrel{o}{x y}_{x}=\frac{3 \sqrt{3}}{2}\left(\mu-\frac{1}{2}\right)+\frac{\sqrt{3}}{2}\left(\frac{15}{2}-\frac{45}{4} \mu+\frac{3 \mu}{1-2 \mu}-\frac{9 \mu}{2(1-\mu)}\right) k_{1}+ \\
& \left(-\frac{\sqrt{3}}{8}-\frac{7 \sqrt{3}}{8} \mu-\frac{2 \sqrt{3} \mu}{1-2 \mu}-\frac{7 \sqrt{3} \mu}{4(1-\mu)}\right) k_{2} \text {, } \\
& \stackrel{o}{\Omega_{y y}}=\frac{9}{4}+\left(6-\frac{33}{8} \mu+\frac{3 \mu}{1-2 \mu}+\frac{21 \mu}{4(1-\mu)}\right) k_{1}+ \\
& \left(\frac{3}{8}+\frac{33}{8} \mu+\frac{3 \mu}{1-2 \mu}+\frac{21 \mu}{4(1-\mu)}\right) k_{2} \text {. }
\end{aligned}
$$

Let $\lambda^{2}=\Lambda$, therefore the characteristic Equation (13) becomes

$$
\left.\Lambda^{2}+\left(4 n^{2}-\stackrel{o}{\Omega_{x x}}-\stackrel{o}{\Omega_{y y}}\right) \Lambda+\stackrel{o}{\Omega_{x x}} \stackrel{o}{\Omega_{y y}}-\stackrel{o}{\left(\Omega_{x y}\right.}\right)^{2}=0
$$

which is a quadratic equation in $\Lambda$. If $\Lambda_{1}$ and $\Lambda_{2}$ are the roots of the Equation (14) then the roots of the characteristic Equation (13) are given by

$$
\lambda_{1,2}= \pm \sqrt{\Lambda_{1}} \text { and } \lambda_{3,4}= \pm \sqrt{\Lambda_{2}}
$$

$\lambda_{i}(\mathrm{i}=1, \ldots 4)$ will be pure imaginary if $\Lambda_{1}$ and $\Lambda_{2}$ both are negative real roots and then the non-collinear libration points will be stable. Now, roots of Eqn. (14) are given by

$\Lambda_{1,2}=\frac{1}{2}\left[\begin{array}{l}-\left(4 n^{2}-\Omega_{x x}-\stackrel{o}{\Omega_{y y}}\right) \pm \\ \sqrt{\left(4 n^{2}-\Omega_{x x}-\stackrel{o}{\Omega_{y y}}\right)^{2}-4\left(\Omega_{x x} \stackrel{o}{\left.\Omega_{y y}-\stackrel{o}{\Omega_{x y}}\right)}\right.}\end{array}\right]$

Eqn. (16) has negative real roots if

$$
\begin{aligned}
& \left.\left(4 n^{2}-\stackrel{o}{\Omega_{x x}}-\stackrel{o}{\Omega_{y y}}\right)^{2}-4 \stackrel{o}{\Omega_{x x}} \stackrel{o}{\Omega_{y y}}-\stackrel{o}{\Omega_{x y}^{2}}\right)=0 \text { i.e. } \\
& 1-27 \mu+27 \mu^{2}+\left(-\frac{195}{2}+261 \mu-\frac{9 \mu}{1-2 \mu}+\frac{39 \mu}{1-\mu}\right) k_{1}+ \\
& \left(-33+\frac{105}{2} \mu-\frac{12 \mu}{1-2 \mu}+\frac{30 \mu}{1-\mu}\right) k_{2}=0
\end{aligned}
$$

For $k_{i}=0(i=1,2)$, the solution of above equation is $\mu=\mu_{o}=$ $0.0385208 \ldots$ (Szebehely, 1967). Now we consider $\mu_{\text {crit }}=\mu_{o}+\alpha, \alpha$ $<<1$ be the solution of Eqn. (17), therefore

$\alpha=\frac{p_{1} k_{1}+p_{2} k_{2}}{2\left(14-27 \mu_{o}\right)}$,

where

$$
\begin{aligned}
& p_{1}=-\frac{195}{2}+261 \mu_{o}-\frac{9 \mu_{O}}{1-2 \mu_{o}}+\frac{39 \mu_{O}}{1-\mu_{O}}, \\
& p_{2}=-33+\frac{105}{2} \mu_{o}-\frac{12 \mu_{O}}{1-2 \mu_{O}}+\frac{30 \mu_{O}}{1-\mu_{O}} .
\end{aligned}
$$

Thus the non-collinear libration points are stable for the critical value of mass parameter $\mu \leq \mu_{\text {crit }}=\mu_{\mathrm{o}}-3.32792 k_{1}-1.16808 k_{2}$.

\section{Conclusion}

In the present paper the existence and stability of non-collinear libration points in restricted three-body problem considering less massive primary an oblate heterogeneous spheroid with N-layers has been discussed and this is found that there exist two noncollinear libration points which are stable for a critical value of mass parameter $\mu \leq \mu_{\text {crit }}=\mu_{\mathrm{o}}-3.32792 k_{1}-1.16808 k_{2}$.

\section{References}

[1] Danby, J.M.A.: Stability of the triangular points in the elliptic restricted problem of three bodies. The Astronomical Journal, 69 (2), 165 - 172 (1964). http://dx.doi.org/10.1086/109254.

[2] Szebehely, V.: Theory of orbits, The Restricted Problem of three bodies. Academic Press, New York and London (1967).

[3] Vidyakin, V.V.: Stability of one particular solution for the motion of three homogeneous spheroids.Soviet Astronomy, 18, 116 (1974).

[4] Sharma, R.K.: Perturbations of Lagrangian points in the restricted three-body problem. Indian Journal of Pure and Applied Mathematics, 6, 1099-1102 (1975).

[5] Subbarao, P.V., Sharma, R.K.: A note on the Stability of the triangular points of equilibrium in the restricted three body problem. Astronomy and Astrophysics, 43, 381-383 (1975).

[6] Sharma, R.K., Subbarao P.V.: Stationary solutions and their characteristic exponents in the restricted three-body problem when the more massive primary is an oblate spheroid. Celestial Mechanics and Dynamical Astronomy, 13, 137-149 (1976). http://dx.doi.org/10.1007/BF01232721.

[7] Choudhary R.K.: Libration points in the generalized elliptic restricted three body problem. Celestial Mechanics, 16, 411 - 419 (1977). http://dx.doi.org/10.1007/BF01229285.

[8] Bhatnagar, K. B., Hallan, P. P.: Effect of perturbed potentials on the stability of Libration points restricted problem. Celestial Mechanics and Dynamical Astronomy, 20(2), 95-103 (1979). http://dx.doi.org/10.1007/BF01230231.

[9] Cid, R., Ferrer, S., Caballero, J.A.: Asymptotic solutions of the restricted problem near equilateral Lagrangian points. Celestial Mechanics and Dynamical Astronomy, 35, 189-200 (1985) http://dx.doi.org/10.1007/BF01227668.

[10] El-Shaboury, S.M.: Equilibrium solutions of the restricted problem of $2+2$ axisymmetric rigid bodies. Celestial Mechanics and Dynamical Astronomy, 50, 199-208 (1991). http://dx.doi.org/10.1007/BF00048764.

[11] Bhatnagar, K.B., Gupta, U., Bharadwaj, R.: Effect of perturbed potentials on the non-linear stability of Libration point L4 in the restricted problem. Celestial Mechanics and Dynamical Astronomy, 59, 45-374 (1994). http://dx.doi.org/10.1007/BF00692102.

[12] Selaru, D., Cucu-Dumitrescu, C.: Infinitesimal orbits around Lagrange points in the elliptic, restricted three-body problem. Celestial Mechanics and Dynamical Astronomy, 61 (4), 333 - 346 (1995). http://dx.doi.org/10.1007/BF00049514.

[13] Markellos, V.V., Papadakis, K.E., Perdios, E.A.: Non-linear stability zones around triangular equilibria in the plane circular restricted three-body problem with oblateness. Astrophysics and Space Science, 245, 157-164 (1996). http://dx.doi.org/10.1007/BF00637811.

[14] Subbarao, P.V., Sharma, R.K.: Effect of oblateness on the nonlinear stability of L4 in the restricted three-body problem. Celestial Mechanics and Dynamical Astronomy, 65, 291-312 (1997).

[15] Khanna, M., Bhatnagar, K.B.: Existence and stability of libration points in restricted three body problem when the smaller primary is a triaxial rigid body. Indian Journal of Pure and Applied Mathematics, 29 (10), 1011-1023 (1998).

[16] Khanna, M., Bhatnagar, K.B.: Existence and stability of Libration points in the restricted three body problem when the smaller primary is a triaxial rigid body and the bigger one an oblate spheroid. Indian Journal of Pure and Applied Mathematics, 30 (7), 721-733 (1999).

[17] Esteban, E. P., Vazquez, S.: Rotating Stratified Heterogeneous Oblate Spheroid in Newtonian Physics. Celestial Mechanics and Dynamical Astronomy, 81, 299 (2001).http://dx.doi.org/10.1023/A:1013292529030.

[18] Roberts, G.E.: Linear Stability of the Elliptic Lagrangian Triangle Solutions in the three-body problem. Journal of Differential Equa-

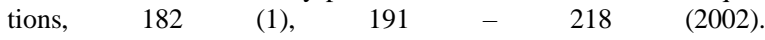
http://dx.doi.org/10.1006/jdeq.2001.4089. 
[19] Oberti, P.,Vienne, A.: An upgraded theory for Helene, Telesto and Calypso. Astronomy and Astrophysics, 397, 353-359 (2003). http://dx.doi.org/10.1051/0004-6361:20021518.

[20] Sosnytskyi, S.P.: On the Lagrange stability of motion in the threebody problem. Ukrainian Mathematical Journal, 57, 1341 - 1349 (2005). http://dx.doi.org/10.1007/s11253-005-0266-8.

[21] Perdiou, A.E., Markellos, V.V., Douskos, C.N.: The Hill problem with oblate secondary: Numerical Exploration. Earth, Moon and Planets, 97, 127-145 (2005). http://dx.doi.org/10.1007/s11038-0069065-y.

[22] John, A. Arredondo, Jianguang, Guo, Cristina, Stoica, Claudia, and Tamayo: On the restricted three body problem with oblate primaries. Astrophysics and Space Science, 341, 315-322 (2012). http://dx.doi.org/10.1007/s10509-012-1085-7.

[23] Idrisi, M. Javed, Taqvi, Z.A.: Restricted three-body problem when one of the primaries is an ellipsoid. Astrophysics and Space Science, 348, 41-56 (2013). http://dx.doi.org/10.1007/s10509-013-1534-y.

[24] Idrisi, M. Javed: Existence and stability of the libration points in CR3BP when the smaller primary is an oblate spheroid. Astrophysics and Space Science, 354, 311-325 (2014). http://dx.doi.org/10.1007/s10509-014-2031-7.

[25] Idrisi, M. Javed, Amjad, Muhammad: Effect of elliptic angle $\varphi$ on the existence and stability of libration points in restricted threebody problem in earth-moon system considering earth as an ellipsoid. International Journal of Advanced Astronomy, 3 (2), 87-96 (2015). http://dx.doi.org/10.14419/ijaa.v3i2.5313.

[26] Idrisi, M. Javed: A general solution to non-collinear equilibria in terms of largest root $(\mathrm{k})$ of confocal oblate spheroid. International Journal of Advanced Astronomy, 4 (1), 01-04 (2016).http://dx.doi.org/10.14419/ijaa.v4i1.5587. 